\title{
RICHARD MILLET DANS LE CONTEXTE LITTÉRAIRE ACTUEL ${ }^{1}$
}

\author{
Ján Drengubiak
}

\begin{abstract}
The article presents a critical overview of the works of Richard Millet who started writing in 1983. Since then, Millet has written a few dozens of books that can be sorted according to several criteria. The subject matter of this paper is to grasp the evolution of the central themes of the author's writings.

Keywords: Richard Millet; author; French contemporary fiction; autobiography.

Résumé : L'article présente un panorama critique de l'œuvre de Richard Millet, auteur français contemporain, dont le premier texte littéraire paraît en 1983. Désormais Millet a écrit plusieures dizaines de livres qui peuvent être étudiés et rangès selon plusieurs critères. Le but de la présentation est de considérer les thèmes clés de l'auteur dans leur évolution.
\end{abstract}

Mots-clés : Richard Millet ; auteur ; roman français contemporain ; autobiographie.

Dans les années $80 \mathrm{du} X \mathrm{X}^{\mathrm{e}}$ siècle, la littérature commence à se poser les questions concernant l'individu, l'histoire et le monde actuel. De ce "renouvellement des questions » (Viart - Vercier 2008 : 25) Bruno Vercier et Dominique Viart font le principe organisateur de la première partie de l'ouvrage La littérature française au présent $(2008)^{2}$ qui vise à cerner les tendances actuelles dans la littérature contemporaine. Ils tracent trois thèmes autour desquels se déroule toute activité romanesque, à savoir le soi, l'histoire et le monde. En effet, chaque questionnement de l'un des thèmes devrait entraîner les deux autres, et même si Millet se méfie des tendances actuelles dans la littérature contemporaine et se veut solitaire : « je n'ai été d'aucune avant-garde, d'aucun mouvement, [...] J'ai toujours été seul. » (Millet 2010a : 73-4) ; il s'inscrit parfaitement dans le cadre de la triple motivation proposée par Viar et Vercier.

L'œuvre de Richard Millet constitue un continuum. D'un côté de ce continuum se situent des romans. Les doubles de l'auteur qui ont plus de cinquante ans et habitent à Paris partagent une expérience d'instituteur ou/et de journaliste avec Millet. Ils ont des ambitions d'écrivains et viennent tous de Siom, village dont le nom évoque Viam en Haute-Corrèze où est né Richard Millet. Viam se trouve dans la partie occidentale du

\footnotetext{
1 This contribution/publication is the result of the project implementation: Retrofitting and Extension of the Center of Excellence for Linguaculturology, Translation and Interpreting supported by the Research \& Development Operational Programme funded by the ERDF.

2 La deuxième partie traite l'évolution des genres et elle n'est pas indispensable pour notre objectif présent.
} 
Massif Central, au Sud-Ouest de Clermont-Ferrand. C'est dans cette région que se déroule l'histoire de plusieurs romans qui conservent la majorité des noms géographiques véritables. Il ne faut pas cependant oublier que les romans sont des « autobiographies transposées » (Millet 2001 : 9), où la saturation en éléments fictifs est la plus élevée. La ressemblance avec la réalité est encore plus manifeste dans les premiers récits de Millet, relatant son expérience libanaise : Beyrouth (1987) et Le balcon à Beyrouth (1994). À propos de l'expérience libanaise Millet dit qu' elle le pousse vers « l'autobiographie directe » (Millet 2004a : 66), néanmoins, la frontière entre la fiction et la vérité ou la réalité y reste toujours incertaine. En tout cas, tous les romans puisent soit dans la matière libanaise oùMillet passe sa jeunesse, ou dans la matière corrézienne où il est né. De l'autre côté de spectre se situent des ouvrages en forme de dialogues. Le premier est « une conversation » avec Chantal Lapeyre-Desmaison, intitulée Fenêtre au crépuscule (2004) qui retrace la vie de Richard Millet dès son enfance. Dans le deuxième ouvrage, Le harcèlement littéraire (2005), Millet répond aux questions de Delphine Descaves et Thierry Cecille, débattant surtout ses orientations intellectuelles. Or, dans le débat surgissent des questions qui exigent une réponse dévoilant des données objectives, et qui expliquent la prédilection pour certains thèmes récurrents dans l'œuvre de l'auteur.

Le premier des grands thèmes de Millet est incontestablement celui de la langue. ${ }^{4}$ Les questions concernant la langue doivent être au moins partiellement résolues avant que l'auteur puisse aborder d'autres thèmes. Toutefois, la langue reste un des fils conducteurs qui traversent toute l'œuvre de l'auteur, tout en conservant un certain degré d'ambiguiité. Cette ambiguïté reflète même la manière dont Millet aborde le thème. Son rapport problématique à la langue est traduit à deux niveaux qui s'entrecroisent : tandis que les essais en portent un témoignage très conscient, les romans et récits repensent les mêmes notions de positions plus indirectes.

Les essais du recueil Le sentiment de la langue rassemblés et publiés pour la première fois en 1988 témoignent de l'affection pour les langues qui se manifeste comme la nostalgie de tout ce qui est inhérent à la langue française, tout ce qui aujourd'hui constitue le «bruissement infini de l'étymologie » (Millet 1993 : 35). Millet déploie cette signification au point de parler parfois au lieu de sentiment, de la « conscience de la langue » ( $c f$. Millet 1993 : 189). Cette conscience de tout ce qui constitue la langue est un acte rationnel par excellence, y compris la conscience « d'une impossible maîtrise, d'un effort perpétuel vers la maîtrise » (Millet 1993 : 189-90) dans toute sa richesse. Cette impossibilité de la maîtrise de la langue est l'enjeu principal qui préoccupe à un degré plus ou moins grand tous les personnages de Richard Millet. Leur prédilection pour ce thème s'établit depuis les premiers romans. Dans les deux premiers, Millet déploie tout un réseau lexical s'attachant à la langue et surtout à la parole. La parole reflète tous les aspects de l'existence des personnages, leurs tempéraments, ainsi que l'humeur qui change d'un moment à l'autre. Parfois, le portrait que le lecteur se fait des personnages est réduit presque exclusivement à leur usage de la langue, voir le conflit principal porte sur la langue. Dans L'invention du corps de Saint Marc (1883), le personnage principal, Marc, cherche un moyen comment guérir sa maladie qui consiste dans le fait qu'il ne peut pas s'exprimer

\footnotetext{
3 La réédition des deux livres apparaît en 2005 sous le titre Le balcon à Beyrouth.

4 La langue dans tous ses aspects est le point de départ par excellence pour un écrivain parce que, comme le dit Barthes, " [1]'écrivain ne peut se définir en termes de rôle ou de valeur, mais seulement par une certaine conscience de parole. Est écrivain celui pour qui le langage fait problème, qui en éprouve la profondeur, non l'instrumentalité ou la beauté » (Barthes 1999: 50).
} 
clairement. La langue est le symptôme de la maladie, mais elle semble être également sa cause ainsi que son remède.

Le roman L'innocence (1984) est la continuation du premier (cf. Millet 2004a : 82) et en tant que tel, le roman développe aussi le motif de la langue. Aloysius, le personnage principal, esclave et prisonnier, est tellement préoccupé par la reproduction fidèle du passé, qu'il vérifie "si l'on n'était point trop mécontent de [son] récit » (Millet 1984: 74) auprès du scribe qui reproduit impassiblement tout ce qu'il relate. Aloysius se rend compte des « contradictions » (Millet 1984 : 11) et la seule possibilité de continuer le récit sans besoin de reconsidérer chaque mot prononcé est de se résigner devant les mots et de se laisser traîner par eux. Aloysius l'explique : «c'était à des mots que je m'abandonnais, dussent-il me mener plus loin que je n'imaginais » (Millet 1984 : 38). A propos de ce roman Richard Millet déclare que c'est « est une réflexion sur le devenir-idiot d'un scripteur : idiot au sens de simple mais aussi d'unique, donc d'innocent » (Millet 2004a : 85). ${ }^{5}$ À l'instant où le parleur et le scribe s'unissent dans la figure de l'écrivain, les ambiguïtés existant entre l'écriture et la parole s'intériorisent. Toutefois, après le roman L'innocence l'écriture ne devient pas immédiatement l'enjeu principal. C'est la parole qui domine encore dans les récits du recueil Sept passions singulières paru un an après, en 1985.

Si dans les premiers textes l'écrivain interroge les moyens d'expression et les limites de la communicabilité esthétique, dans L'angélus (1988), La chambre d'ivoire (1989), L'écrivain Sirieix (1992) Millet aborde l'acte d'écrire (cf. Coyault-Dublanchet 2002 : 75). Cela ne signifie point qu'il exclut désormais tout discours sur la langue, mais celui-ci n'est plus l'enjeu principal. Les trois romans de la petite trilogie noire se prêtent à une lecture qui les réunit autour du thème de la création artistique et de la condition d'artiste. Tout d'abord, Millet traite la question de l'impossibilité d'une œuvre et la complémentarité entre la souffrance et le plaisir dans L'angélus ; ensuite, il questionne les conditions de la création, à savoir, le besoin de la réclusion, du silence et de l'immobilité dans La chambre d'ivoire ; finalement, il s'interroge sur la formation nécessaire de l'écrivain dans L'écrivain Sirieix. Trois récits, trois échecs, les trois romans sont marqués par le même désir de sincérité des narrateurs, de dire leur imposture, le mensonge de leur vie, la fausseté de leur obsession de l'Euvre. Ce sont trois romans du paradoxe insoluble de la condition d'artiste. La nature des paradoxes est telle que toute réponse porte en elle l'élément de sa contradiction et que, dans ce sens, de véritables réponses ne sont pas possibles. C'est pour cette raison que le motif de la condition de l'artiste ne disparaît plus de l'œuvre. Néanmoins, l'importance de la trilogie réside dans le fait qu'elle rend possible la continuation du travail de l'écrivain, voire sa nécessité, parce que, même si tout art est imposture, c'est dans sa vie qu'il faut se délivrer du mensonge. En plus, à l'instant où Millet résout partiellement des problèmes qui le hantent en tant qu'artiste, il peut porter son regard sur la culture qui l'entoure. C'est dans le projet de la survie de la culture qu'il trouve sa véritable vocation, sous-entendue dans la phrase « tout écrivain porte en lui la mort du monde et sa résurrection » (Millet 2007a : 27).

Bien avant d'exprimer explicitement la conviction qu' on peut faire renaître toute une culture en racontant sa mort dans la littérature, les romans qui accomplissent ce projet sont déjà écrits. En effet, après les années que Richard Millet a investies dans l'élucidation des ambiguïtés concernant la langue et la création artistique, les préoccupations per-

5 Cette « rupture avec le social » (Millet 2004a : 85) s'accomplit encore comme l'emprisonnement d'Aloysius, tandis que plus tard la rupture devient le choix conscient des personnages de s'exiler. 
sonnelles cèdent la place à un projet plus ambitieux : « amener [le roman] à l'universalité du littéraire » (Millet 2010a : 275). Ce tournant important survient avec l'avènement des textes puisant dans la matière limousine. Même si la référence à la région apparaît déjà dans certains récits du recueil Cour blanc (1994) et dans la petite trilogie noire, c'est le roman La gloire des Pythre (1995) qui situe l'histoire à Siom en Corrèze et devient le premier tome de la «trilogie siomoise ". $^{6}$ À première vue, la transition n'est point abrupte, parce que les thèmes partiels déjà introduits ne disparaissent jamais de l'œuvre, c'est seulement l'importance qui leur est accordée, qui change. Les innovations s'établissent progressivement, mais à l'instant où la matière limousine est déployé avec vigueur, elle devient porteuse de l'universel. Pour ce faire, Millet recourt au mythe ce qu'il dit aussi explicitement : «La province, c'est le lointain ayant eu accès à l'intemporel par excès de localisation - ce qui est une prodigieuse source de romanesque mais qui a besoin d'une forme de damnation pour atteindre au mythe » (Millet 2010a : 152). Il constate aussi qu'en Corrèze il vivait « au sein d'un grand récit mythologique, ou plus exactement en un récit en quête de son propre légendaire, lequel n'est sans doute qu'une perpétuelle redéfinition de lui-même » (Millet 2011a : 50). Les romans de la trilogie siomoise, La gloire des Pythre, L'amour des trois sœurs Piale (1997), Lauve le pur (2000), ainsi que le roman que Millet écrit plus tard, Ma vie parmi les ombres (2003), racontent l'histoire de la civilisation qui est en train de disparaître avec toutes ses valeurs et ses traditions. Afin que le souvenir survive, il est indispensable de le transmettre dans sa totalité. Dans ce but, l'auteur imagine plusieurs narrateurs et perspectives. Afin de donner aux romans et au passé qui en est le thème une portée universelle, l'auteur recourt de plus en plus explicitement à l'imaginaire mythique.

Même si la mort de la civilisation et de la culture fait de longtemps partie intégrante des réflexions de Millet, les implications que cette mort entraîne pour l'avenir de la société, sont relativement récentes dans l'œuvre. Cette considération prend - comme d'habitude - deux formes différentes. Explicite, consciente et préméditée dans les essais, elle devient plus implicite, allusive et en fin de compte plus convaincante dans les romans et récits. Dans les romans le rôle d'observer la décadence de perspectives diverses, est confié aux narrateurs. Si Thomas Lauve observe la fin de la civilisation depuis son centre à Paris il voit une décadence générale dans le contexte mondial, mais les nuances lui échappent. Les narratrices des romans Le cavalier siomois (2004) et Dévorations (2007), en revanche, adoptent un regard particulier, féminin, celui de la périphérie. Leur perspective dévoile ce dont Thomas ne pourrait jamais témoigner d'une manière convaincante. Malgré les perspectives différentes, dans chacun des textes, l'image de l'échec de la communauté à trouver les moyens de perpétuer ses valeurs et traditions est exploitée sur plusieurs plans. Sur le plan des histoires individuelles des narrateurs, sur le plan de la désintégration de la famille autant que sur le plan symbolique. En tout cas, la civilisation rurale semble être voué inévitablement à la disparition. Et si dans la fin de la civilisation rurale, Millet voit « le prodrome de la fin de la civilisation européenne, donc de l'humanisme » (Millet 2011a : 49), il dessine ainsi une image sombre de l'avenir.

Dans les œuvres les plus contemporaines, on remarque chez Millet une tendance à revenir aux essais et réflexions personnelles traitant des sujets aussi divers que dans le Sentiment de la langue: Musique secrète (2004), La voix et l'ombre (2012) sont inspirés par

\footnotetext{
6 Il est nécessaire de distinguer entre « la trilogie siomoise » qui situe les romans à Siom et « la matière limousine » ou « corrézienne » qui a pour cadre toute la région, Siom y compris.
} 
la musique. Le dernier écrivain (2005), Harcèlement littéraire (2005), Désenchantement de la littérature (2007) L'opprobre, Essai de démonologie (2008), L'enfer du roman : Réflexions sur la postlittérature (2010) portent surtout sur la situation de la littérature contemporaine. Fatigue du sens (2011), Arguments d'un désespoir contemporain (2011) développent les réflexions de L'enfer du roman dans le domaine plus général de la culture, tradition, modernité, tandis que Petit éloge d'un solitaire (2007) et L'amour mendiant : Notes sur le désir (2007) partagent les méditations plus intimes. Vers la fin de la même décennie, on voit également un retour à la matière libanaise qui semblait épuisée en 1994 par Le balcon à Beyrouth. Le thème revient avec une telle insistance qu'à partir de 2009 quand paraît La confession négative et L'Orient désert, Millet publie plusieurs récits et romans Brumes de Cimmérie (2010), Le sommeil sur les cendres (2010), Cinq chambres d'été au Liban (2010) ou La fiancée libanaise (2011). Et il ne faut point oublier les trois textes datant de 2012 qui ont suscité un débat passionné autour de l'auteur et ont valu son poste d'éditeur chez Gallimard : De l'antiracisme comme terreur littéraire, Intérieur avec deux femmes et Langue fantôme suivi d'éloge littéraire d'Anders Breivik. Enfin, quelle sera la dirrection suivante que va prendre l'œuvre de Richard Millet semble être une question ouverte.

\section{Bibliographie}

BARTHES, Roland (1999), Critique et vérité, Paris : Éditions du Seuil (première édition 1966).

Coyault-Dublanchet, Sylvianne (2002), La Province en héritage, Genève : Droz.

Millet, Richard (1983), L'invention du corps de Saint Marc, Paris : P.O.L.

Millet, Richard (1984), L'innocence, Paris : P.O.L.

Millet, Richard (1985), Sept passions singulières, Paris : P.O.L.

Millet, Richard (1993), Le sentiment de la langue, Paris : La table ronde.

Millet, Richard (1994), Cour blanc, Paris : P.O.L.

Millet, Richard (1995), La gloire des Pythre, Paris : Éditions Gallimard.

Millet, Richard (1997), L'amour des trois scurs Piale, Paris : Éditions Gallimard.

Millet, Richard (2000), Lauve le Pur, Paris : Éditions Gallimard.

Millet, Richard (2001), L'angélus - La chambre d'ivoire - L'écrivain Sirieix, Paris: Éditions Gallimard (édition citée).

Millet, Richard (2003), Ma vie parmi les ombres, Paris : Éditions Gallimard.

Millet, Richard (2004a), Fenêtre au crépuscule, Paris : La table ronde.

Millet, Richard (2004b), Le cavalier siomois, Paris : La table ronde.

Millet, Richard (2005a), Le dernier écrivain, Saint-Clément-de-Rivière : Fata Morgana.

Millet, Richard (2005b), Le balcon à Beyrouth, Paris : La table ronde.

MiLLET, Richard (2005c), Le harcèlement littéraire, Paris : Éditions Gallimard.

Millet, Richard (2007a), Désenchantement de la littérature, Paris : Éditions Gallimard.

Millet, Richard (2007b), Dévoration, Paris : Éditions Gallimard.

Millet, Richard (2007c), Petit éloge d'un solitaire, Paris : Éditions Gallimard.

Millet, Richard (2008), L'opprobre, essai de démonologie, Paris : Éditions Gallimard.

Millet, Richard (2010a), L'enfer du roman. Réflexions sur la postlittérature, Paris: Éditions Gallimard.

Millet, Richard (2010b), Tarnac, Paris : Gallimard/L'Arpenteur.

Millet, Richard (2011a), Arguments d'un désespoir contemporain, Paris : Hermann.

Millet, Richard (2011b), Fatigue du sens, Paris : Pierre Guillaume de Roux.

Millet, Richard (2004), Musique secrète, Paris : Éditions Gallimard. 
MiLlet, Richard (2012), La voix et l'ombre, Paris : Éditions Gallimard.

Millet, Richard (2012), De l'antiracisme comme terreur littéraire, Paris: Pierre Guillaume de Roux.

Millet, Richard (2012), Intérieur avec deux femmes, Paris : Pierre Guillaume de Roux.

MiLlet, Richard (2012), Langue fantôme suivi d'éloge littéraire d'Anders Breivik, Paris : Pierre Guillaume de Roux.

Millet, Richard (2009), La confession négative, Paris : Éditions Gallimard.

MilLET, Richard (2009), L'Orient désert, Paris : Mercure de France.

Millet, Richard (2010), Brumes de Cimmérie, Paris : Éditions Gallimard.

Millet, Richard (2010), Le sommeil sur les cendres, Paris : Éditions Gallimard.

Millet, Richard (2010), Cinq chambres d'été au Liban, Paris : Éditions Gallimard.

Millet, Richard (2011), La fiancée libanaise, Paris : Éditions Gallimard.

VIART, Dominique - VERCIER, Bruno (2008), La littérature française au présent ; Héritage, modernité, mutation. ( $2^{\mathrm{e}}$ édition augmentée), Paris : Bordas.

\author{
Ján Drengubiak \\ Katedra francúzskeho jazyka a literatúry \\ Inštitút románskej a klasickej filológie \\ Filozofická fakulta \\ Prešovská univerzita \\ Ul. 17. novembra 1 \\ 08078 Prešov \\ Slovaquie \\ drengubiak@yahoo.com
}

